\title{
Improving groundwater-flow modeling using optimal zoning methods
}

\author{
Ching-Pin Tung $\cdot$ Chung-Che Tang $\cdot$ Yu-Pin Lin
}

\begin{abstract}
Hydraulic conductivity sometimes exhibits complicated spatial variation over a site. A thorough understanding of the spatial distributions of hydraulic conductivity helps to make deterministic models of groundwater more accurate. This study presents a novel procedure that combines simulated annealing algorithms (SA) and the shortest distance method (SD) with the modular three-dimensional groundwater flow model (MODFLOW). The procedure is applied to a hypothetical site with groundwater-monitoring wells to minimize the difference between simulated and observed hydraulic head for optimal zoning of the spatial distribution of hydraulic conductivity. The results of this optimal zoning method indicate that this new procedure not only improves the efficiency of optimization, but also increases the probability of finding the global optimum, minimizing the errors of the hydraulic head simulated by MODFLOW in two scenarios, one with known and the other with unknown hydraulic conductivity. The results also illustrated that the procedure can effectively determine and delineate hydrogeological zones.
\end{abstract}

Keywords Hydraulic conductivity - Hydraulic head - Optimization - Shortest distance method $\cdot$ Simulated annealing algorithms

Received: 4 November 2002 / Accepted: 7 April 2003

Published online: 8 July 2003

(C) Springer-Verlag 2003

\section{C.-P. Tung}

Department of Bioenvironmental Systems Engineering, National Taiwan University, Taipei, Taiwan

C.-C. Tang

Department of Bioenvironmental Systems Engineering,

National Taiwan University, Taipei, Taiwan

Y.-P. Lin ( $\square)$

Department of Landscape Architecture, Chinese Culture University, 55 Hwa-Ken Rd. Yangming Shan, 11114, Taipei, Taiwan

E-mail: yplin@faculty.pccu.edu.tw

Tel.: +886-2-28626433

Fax: +886-2-28617507

\section{Introduction}

Groundwater simulation is an essential task in groundwater management. Accurate simulations of groundwater provide information required to manage groundwater resources and regional water resources. Models of groundwater simulation, such as the modular three-dimensional groundwater flow model (MODFLOW), developed by the USGS (United States Geological Survey; McDonald and Harbaugh 1988), have been developed to numerically solve the groundwater equation to simulate groundwater flow. In MODFLOW, the study area must be discretized into cells to which parameters are assigned to simulate confined or unconfined flow and saturated flow, in one, two, or three dimensions, using finite-difference techniques. Hydraulic conductivity is the parameter that most strongly influences groundwater simulation. This parameter sometimes contains significant levels of uncertainties, including complex (unexplainable) variations in observed values of measurable attributes over investigated area (Lin and others 2001b). A thorough understanding of the spatial distributions of hydraulic conductivity helps to construct more accurate deterministic models of groundwater. The reconstruction of the hydraulic conductivity field from the more numerous experimental hydraulic head data, an inverse problem, raises the issue not only of the complexity of the diffusion equation that links the two variables, but also of the need to account for the physical aspects of the site under study, including, for example, the boundary conditions, the effective recharge, and the geology (Roth and others 1998). Different kinds of methods, such as inverse and kriging methods, exist for determining hydrogeological parameters as inputs to models of groundwater. The hydrogeological parameters can be estimated using these methods with trial and error, iteration, or optimization techniques. Examples are presented in Wang and Zheng (1998), Roth and others (1998), Sophocleous and others (1999), Wen and others (1999), Wingle and others (1999), Abdulla and others (2000), Mikake and others (2000), Lin and others (2001b), Detwiler and others (2002), Ella and others (2002), Majumdar and others (2002), and Tung and Chou (2002).

The simulated annealing algorithm (SA) is a probabilistic hill-climbing algorithm that has received much attention. Unlike other optimization methods, SA can escape from a local optimum, increasing the probability of reaching the global optimum. It is appropriate for multi-dimensional 
optimization programming. The basic concept of SA was first used in computer-simulation methods in condensed matter physics, as described by Metropolis and others (1953). Kirkpatrick and others (1983) then successfully applied the method to combinatorial optimization problems.

This study develops an optimal zoning procedure for applying SA and the shortest distance (SD) method with MODFLOW to determine the best zonation of hydraulic conductivity. During optimization, the difference between the simulated and the observed hydraulic head is minimized. Moreover, comparisons of optimization results between the proposed optimal zoning method and the newer version of MODFLOW with the Gauss-MarquardtLevenberg method of nonlinear parameter estimation are also addressed in this paper.

\section{Materials and methods}

This study considers a hypothetical case that involves a simplified version of a real area to prove the feasibility of this new algorithm. A hypothetical confined aquifer with known groundwater withdrawals, zonation of hydraulic conductivity, and specific storage coefficients is considered. The area is divided into $1212 \times 2-\mathrm{km}$ cells, as shown in Fig. 1. In this aquifer, the north, south, and west bounds are constant head boundaries, and the east bound is a constant flux boundary. The depth of the aquifer is $30 \mathrm{~m}$. Tables 1 and 2 state designed hydraulic conductivity, specific storage coefficients, and the monthly pumpings, respectively. For simplicity, in this case, the slight vertical recharge was ignored.

This study considers two scenarios to test this new algorithm. Practically, the hydraulic conductivity of an area can only be obtained from a limited number of hydrogeologic observation wells distributed in the area. Accordingly, the spatial distributions of hydraulic conductivity are unknown. A designed area with zoned hydraulic conductivity is assumed to be known and the zoning is also assumed to be optimum to solve the problem. Thus, the hydraulic head is the observed head, according to zoning. Figure 1 depicts the designed area with an assumed zoning of hydraulic conductivity. The dotted cells in Fig. 1 are the locations of nine groundwater monitoring wells. In the first scenario, the hydrogeologic observation wells are within the study area and the hydraulic conductivity in this area can be obtained from these wells to verify the proposed method. The number of zones is assumed to be known and equal to the number of observation wells. However, the spatial distribution of hydraulic conductivity is still unknown. Thus, this type of hydraulic conductivity in this area can be determined from these wells, helping to optimize zoning.

In the second scenario, hydrogeologic observation wells within the study area are lacking and the type of hydraulic conductivity is unknown. Then, an iterative procedure is applied to determine the number of types of hydraulic conductivity (i.e., the number of zones). At first, two types are considered to optimize the spatial distribution of hydraulic conductivity; the next trial involves three types and so on, until the optimized spatial distribution of hydraulic conductivity is found.

\section{Simulated annealing algorithms (SA)}

The simulated annealing algorithm is based on the analogy between the annealing of a solid and the optimization of a system with several independent variables. A solid is annealed by raising its temperature such that its particles become randomly arranged in the liquid phase, and then cooling it forces the particles into the low energy states of a regular lattice. At high temperatures, all possible states can be reached, although low energy states are occupied with a higher probability; the cooling process reduces the number of accessible states and the system is finally frozen in its ground state. As in an optimization process, a system may initially have one of several different configurations.
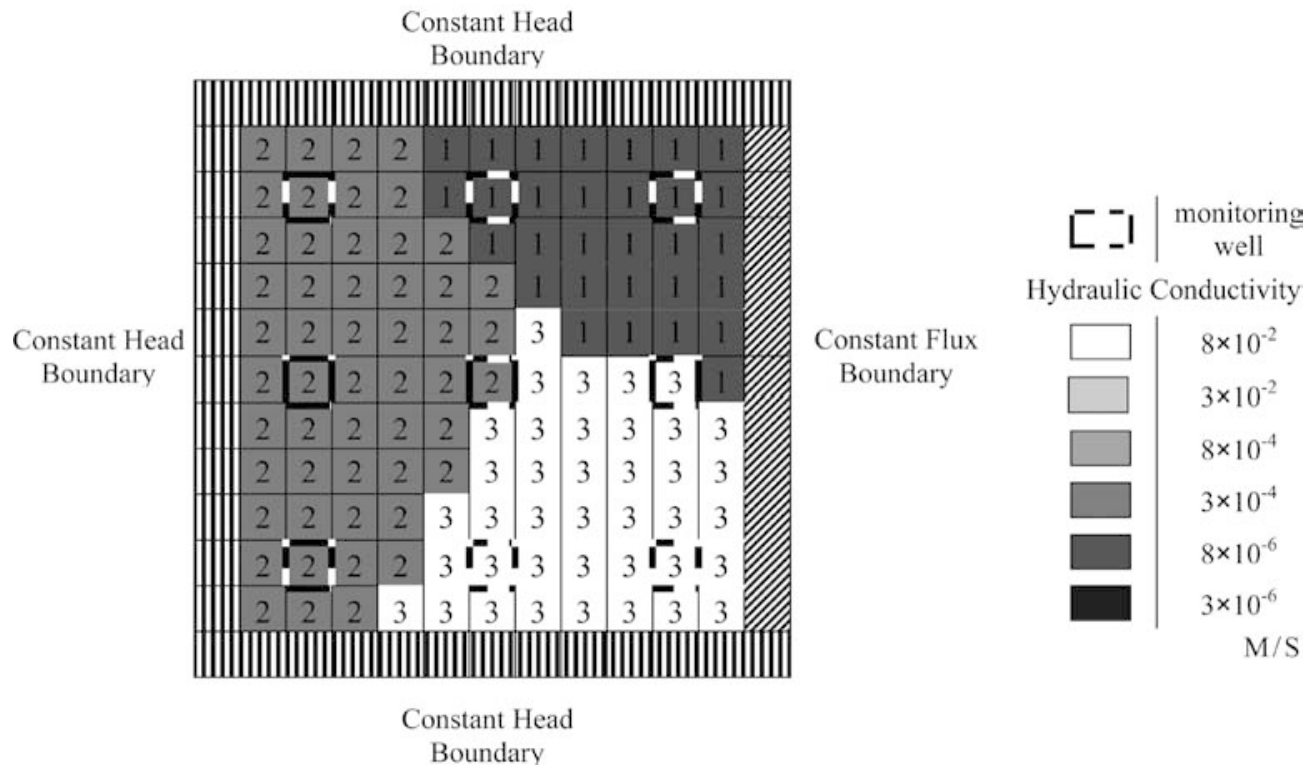

Fig. 1

The zonation in a simulated area 
Table 1

Hydraulic conductivities and specific storage coefficients for designed aquifer

\begin{tabular}{|c|c|c|}
\hline & $\begin{array}{l}\text { Hydraulic conductivity } \\
(\mathrm{m} / \mathrm{s})\end{array}$ & $\begin{array}{l}\text { Specific storage coefficient } \\
(1 / \mathrm{m})\end{array}$ \\
\hline 1 & 0.03 & 0.0004 \\
\hline 2 & 0.0008 & 0.0006 \\
\hline 3 & 0.000003 & 0.0001 \\
\hline
\end{tabular}

Table 2

Designed monthly groundwater pumping for all grids (unit: $\mathrm{cm}$ )

\begin{tabular}{lc}
\hline Month & Groundwater pumping \\
\hline 1 & 0.0040 \\
2 & 0.0040 \\
3 & 0.0035 \\
4 & 0.0030 \\
5 & 0.0030 \\
6 & 0.0025 \\
7 & 0.0020 \\
8 & 0.0015 \\
9 & 0.0020 \\
10 & 0.0030 \\
11 & 0.0040 \\
\hline
\end{tabular}

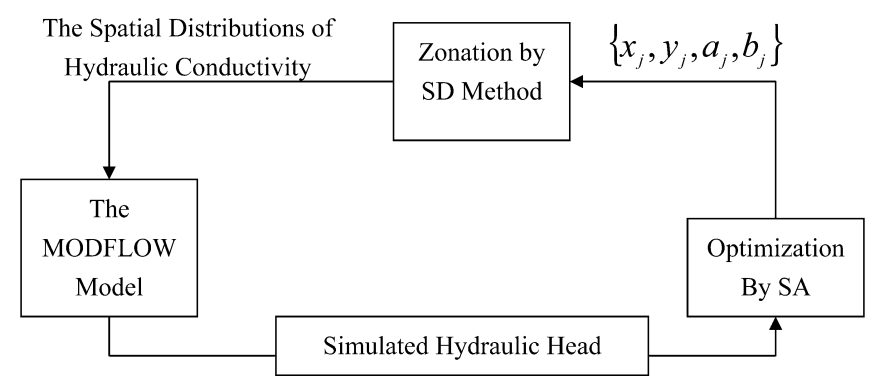

Fig. 2

Flowchart of SA

As in cooling, the search for a configuration converges on an optimal state. Figure 2 shows the flowchart of SA.

\section{Initial and neighbor solution}

Generally, the initial solution is selected randomly or by following some rules. Optimization begins at the initial solution and different initial solutions may lead to different local optimal solutions in a nonlinear problem. However, a robust algorithm must yield a minimally deviating optimum from various initial solutions.

All the neighbor solutions establish a neighborhood. The core of SA is the criterion for accepting a neighbor solution. Unlike general algorithms, SA has a probability of accepting a poor neighbor solution. Accordingly, the optimization process is prevented from becoming stuck at a local optimum, enabling it to find a global minimum.

\section{Acceptance criterion}

Whether a move should be made from a current solution to a neighbor one is judged by an acceptance criterion.
Allow $E_{c}$ and $E_{n}$ to denote the energy of the current and neighbor solutions, respectively. If $E_{c}>E_{n}$, then a lower energy has been reached, and the neighbor solution is accepted and becomes the current one. However, if $E_{t} \geq \mathrm{E}_{c}$ then the neighbor solution is accepted as the current one with a probability that is proportional to a temperature function (the Boltzmann distribution).

$D=\exp \left[-E_{t}-E_{c} / T\right]$

The above temperature function in simulated annealing procedure controls how fast the optimization function is reduced by allowing some switches that increase the optimization function (Deutsch and Journel 1992; Lin and others 2001a). The temperature parameter, $T$, in Eq. (1) is analog to the annealing procedure (Lin and others 2001a), which is nothing to do with real groundwater temperature. The higher the temperature, the greater the probability that an unfavorable swap will be accepted (Deutsch and Journel 1992; Lin and others 2001a).

During searching for optimization solution, the temperature parameter, $T$, is gradually reduced. The cooling schedule also influences the convergence of the SA. The parameters of the schedule are (1) the length of the Markov chains (fixed number of iterations), (2) the initial temperature, (3) the decrement in temperature, and (4) the stop criterion. The following subsection considers the formal aspects of the schedule. A well-set cooling schedule leads to good optimization.

1. Length of the Markov chains (the number of iterations). At each temperature, the number of iterations $L$ strongly influences the result of the optimization. Generally, the number of iterations should be large enough to enable the system to reach a steady state. Kirkpatrick and others (1983) suggested that the number of iterations should be much more times of decision variables.

2. The initial temperature. The initial temperature $T_{i}$ should be high enough to ensure that initially all solutions occur with equal probability. This requirement is necessary to prevent the optimization process from becoming stuck at a local minimum. Previous researchers have found that the initial temperature $T_{i}$ should be selected to yield an initial acceptance rate of at least $80 \%$ (Kirkpatrick and others 1983). Dougherty and Marryott (1991) also suggested that choosing $T_{i}$ too high is preferable to choosing it too low, to ensure thermal equilibrium conditions.

3. Decrement of temperature. The temperature $(T)$ at step $n$ is related to $T$ at step $n-1$ by $T_{n}=\alpha \mathrm{T}_{n}{ }^{-1}$, where $\alpha$ is a temperature decrement coefficient that satisfies $0<\alpha<1$. Traditionally, a constant $\alpha$, usually chosen between 0.8 and 0.99 , is used throughout the cooling process (Kirkpatrick and others 1983; Kirkpatrick 1984). The minimum value of $\alpha=0.5$ ensures sufficiently slow cooling at high $\mathrm{T}$.

4. Stop criterion. The cooling process terminates when the system is frozen, i.e., when the value of the cost function of the solution does not improve after a certain 
number of temperature drops (Dougherty and Marryott 1991). The cooling process can also be stopped as soon as the required solution is found.

The best solution found during the process is compared with the best recorded solution to ensure that the optimization process converges to the global optimum. The best recorded solution is replaced by any better solution.

\section{Shortest distance method}

The shortest distance method (SD) was applied to decide the zoning of a hydrogeologic parameter. The presented SD method involves an area divided into cells on a grid, $g_{i j}\left(x_{i}, y_{j}\right)(i=1,2, \ldots, m ; j=1,2, \ldots, n)$. The zonation is determined by the location of the representative cells, known as Medoids (Kaufman and Rousseeuw 1990), obtained by the following steps:

- Step 1: assume that the cells $g_{i j}\left(x_{i}, y_{j}\right)$ are assigned to $c$ zones, $Z_{k}, k=1, \ldots, c$. Initially, as many medoids $M_{k}\left(x_{i}\right.$, $\left.y_{j}\right), k=1,2,3, \ldots, c$ are selected randomly.

- Step 2: compute the Euclidean distance $d_{i j}$ between each cell $i$ and each medoid $j$. The membership is obtained from the Euclidean distance.

$$
\begin{aligned}
& d_{i j}=\sqrt{\left(g_{i j}-M_{k}\right)^{2}} \quad 1 \leq i \leq m, 1 \leq j \leq n, 1 \leq k \leq c \\
& W_{i j}=\frac{1}{d_{i j}}
\end{aligned}
$$

Step 3: analyze the membership of a cell and consider to which medoid the cell is assigned. A cell should be assigned to the medoid with the highest membership or the nearest medoid.

Step 4: repeat steps 1 to 3 until all cells are analyzed and the zones are defined.

For example, consider the square shown in Fig. 3 for $m=n=11, c=3$. Following the above steps, three medoids are selected randomly and shown as the broader cells. Accordingly, zones are defined successfully. Cells in the same zone have the same hydrogeologic characteristics as their medoid.

The SD method can define a zone only by determining medoids. Thus, the number of variables increases with the number of zones. Three variables are created when a zone is added.

The SD method applies the shortest distance concept just like the $K$-mean algorithm and the Thiessen's method.

However, the $K$-mean algorithm iteratively computes the optimal centroids of clusters. The Thiessen method defines zones using constant centroids, such as known hydrogeologic observation wells. However, the latter method is limited by any lack of hydrogeologic observation wells. In the SD method, no hydrogeologic observation wells is required, the spatial distribution of hydrogeologic parameter is defined only by the determining medoids and their hydrogeologic characteristics. The optimal medoids in zoning may provide the justification for building hydrogeologic observation wells.

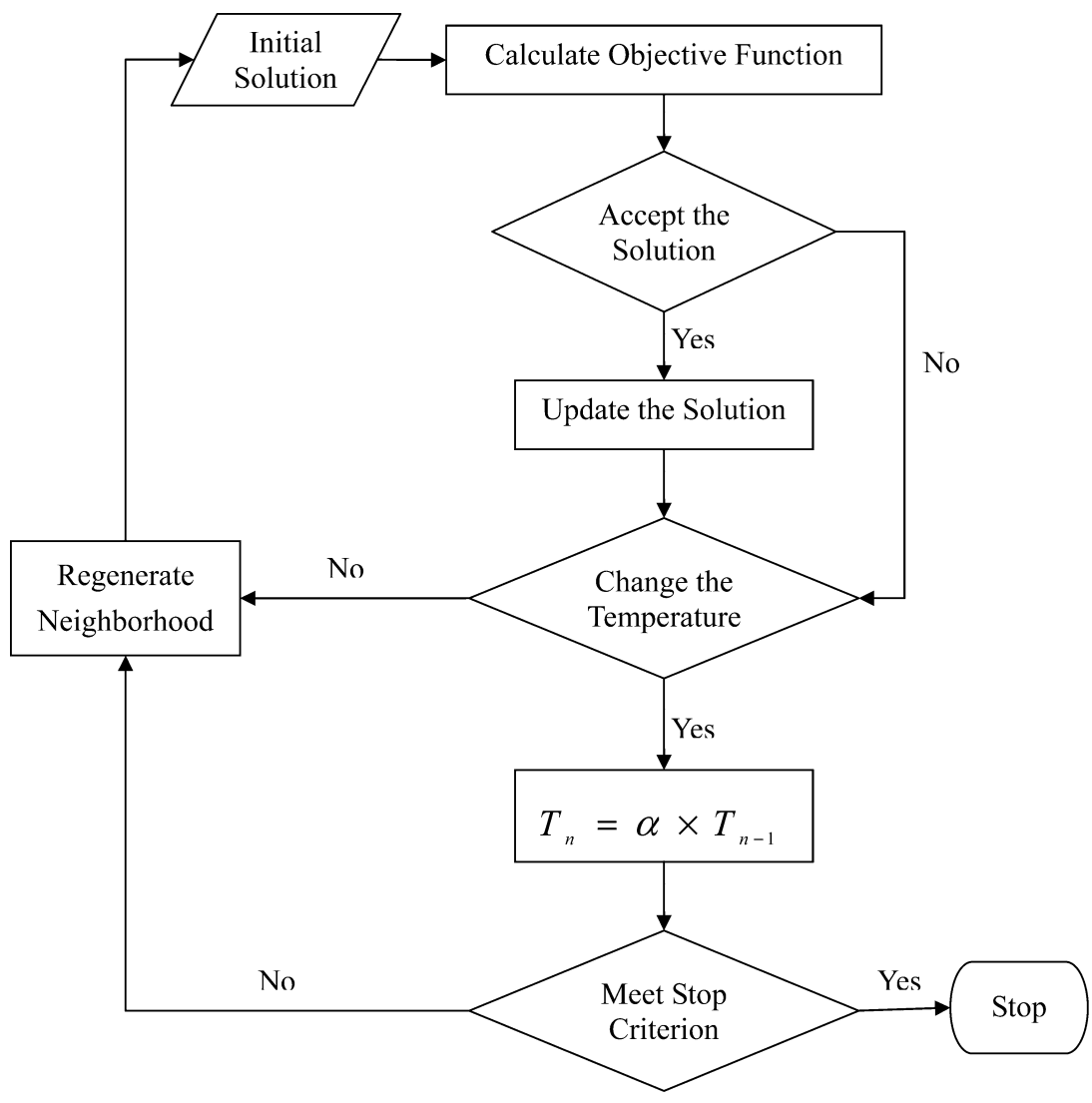

Fig. 3 Zonation of the shortest distance method 


\section{Formulation of the optimal zonation problem}

The global optimal zonation is designed and, thus, known in the case considered. Thus, the difference between the simulated and the observed head, which is adopted as the objective function, is expected to be zero. The zonation problem is mathematically modeled as follows.

Min

$$
Z=\sum_{i, t}\left|h^{c}(i, t)-h^{o}(i, t)\right| \quad i=1,2,3 \cdots n
$$

S.T.

$T s \frac{\partial^{2} h}{\partial x^{2}}+T s \frac{\partial^{2} h}{\partial y^{2}}-W=S \frac{\partial h}{\partial t}$

$x_{l} \leq x \leq x_{u}$

$y_{l} \leq y \leq y_{u}$

where $h^{c}(i, t)$ and $h^{o}(i, t)$ are the calculated and observed hydraulic heads at time $t$ and in cell $i$ that includes a groundwater observation well; $n$ is the number of groundwater observation wells; Ts is the transmissivity defined as the product of the horizontal conductivity and the thickness of the aquifer; $S$ is specific yield, and $w$ is the net vertical recharge. The terms $x_{l}, x_{u}, y_{l}, y_{u}$ are the lower and upper bounds of the medoid coordinates. In the study, $x_{l}, y_{l}$ is set to 1 and $x_{u}, y_{u}$ is set to 11 . Each step by which the medoids move is two cells long. As well as $x$ and $y$, hydraulic conductivity is also a decision variable. The aquifer is assumed to be made of fine sand and the hydraulic conductivity between $10^{-2}$ and $10^{-6}$ is set to $K=a \times 10^{-b}$, where $a \in\{3,5\}$ and $b \in\{2,4,6\}$. Accordingly, the hydraulic heads can be solved using the MODFLOW model (McDonald and Harbaugh 1988). The term $w$ and the spatial distributions of $K$ and $S$ are given inputs. The decision variables of this optimization problem can then be defined as $\left\{x_{j}, y_{j}, a_{j}, b j\right\}$.

\section{Specification of SA}

Before SA is applied to optimize the zoning, some information must be given, including initial zoning, neighborhood, and cooling schedule.

\section{Initial zoning and neighborhood}

The initial zoning of the entire search process is determined by randomly selecting medoids. The zoning is given by the coordinates of the medoids.

This study applied a rule that a variable is changed stepwise to define a neighborhood. For example, a solution set $\left\{x_{j}, y_{j}, a_{j}, b_{j}\right\}$ may have a neighborhood of eight solution sets.

\section{Cooling schedule}

The parameters of the cooling schedule set here are case independently.

1. Length of the Markov chains (the number of iterations). According to Kirkpatrick and others (1983) the number of iterations $L$ is set to several times of the number of decision variables $\left\{x_{j}, y_{j}, a_{j}, b_{j}\right\}$.

2. Initial temperature. Following the suggestion that the initial temperature $T_{i}$ should yield an initial acceptance rate of at least $80 \%$ (Kirkpatrick and others 1983), the initial temperature is set in this study to 10 .

3. Decrement of temperature. In this study, the value of 0.8 is assigned to the decrement of temperature (Kirkpatrick and others 1983).

4. Stop criterion. In this study, the stop criterion is set when the value of the cost function of the solution does not improve after a certain number of temperature decrement (Dougherty and Marryott 1991). The optimization process is also stopped as soon as the global optimum is found.

Figure 4 presents the optimization procedure, which is described as follows. (1) An initial zonation is given and the spatial distribution of hydraulic conductivities is determined accordingly. (2) Then, the value of the objective function can be calculated after the MODFLOW model is run to simulate hydraulic heads. (3) A neighborhood can be determined by current solution. (4) The objective values of neighbor solutions can also be evaluated. (5) The step of stopping moves to the best solution, which meets the stopping criterion of SA. (6) If the criterion to stop searching is not met, go to step (3). Otherwise, the recorded solution is the found optimal solution.

\section{Results and discussion}

\section{Scenario 1}

The global optimal spatial distributions of hydraulic conductivity are described above. Three types of hydraulic conductivity are known. Five randomly selected zonings are used to initiate the optimization, as shown in Fig. 5. The difference between simulated and observed head is to be minimized. The results reveal that given these five trials, the global optimum is found

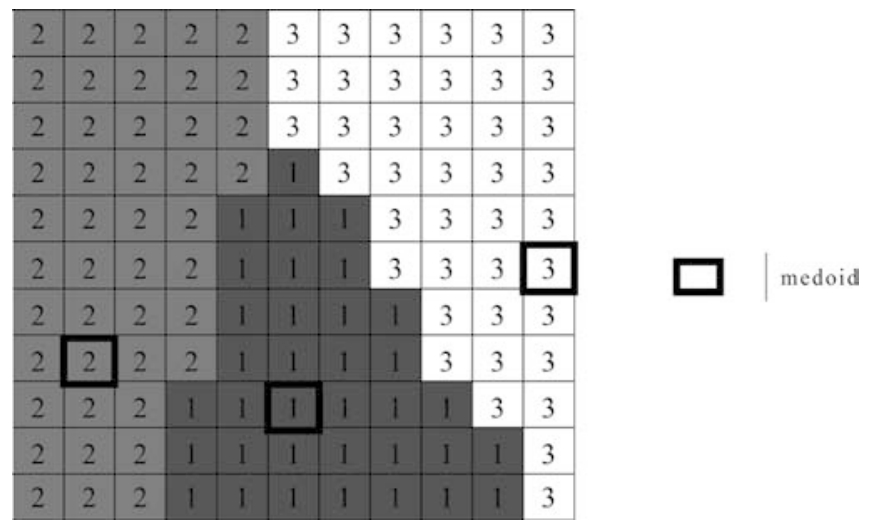

Fig. 4

Flowchart of the optimization procedure 

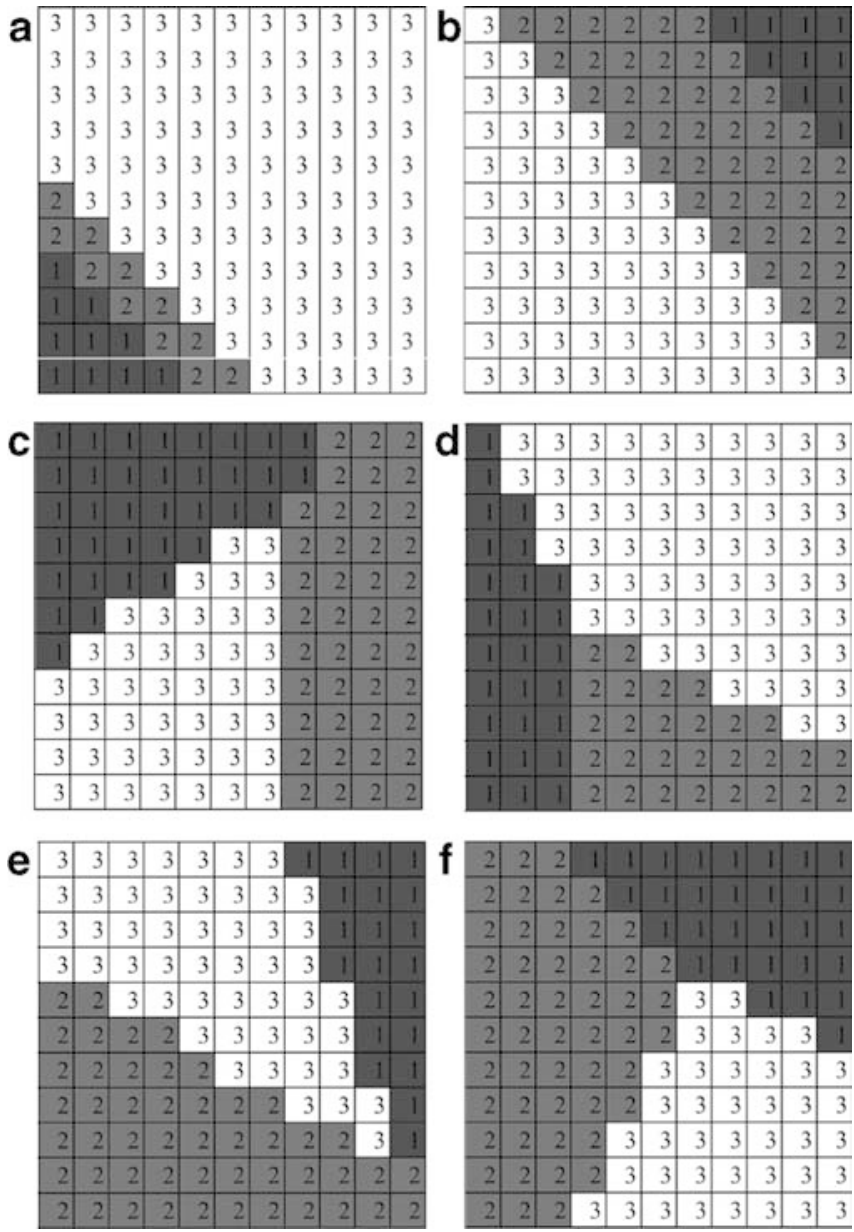

Fig. 5

a The initial zonation of the first trial; $\mathbf{b}$ the Initial zonation of the second trial; $\mathbf{c}$ the initial zonation of the third trial; $\mathbf{d}$ the initial zonation of the fourth trial; $e$ the initial zonation of the fifth trial; $f$ the final zonation of the fourth trial
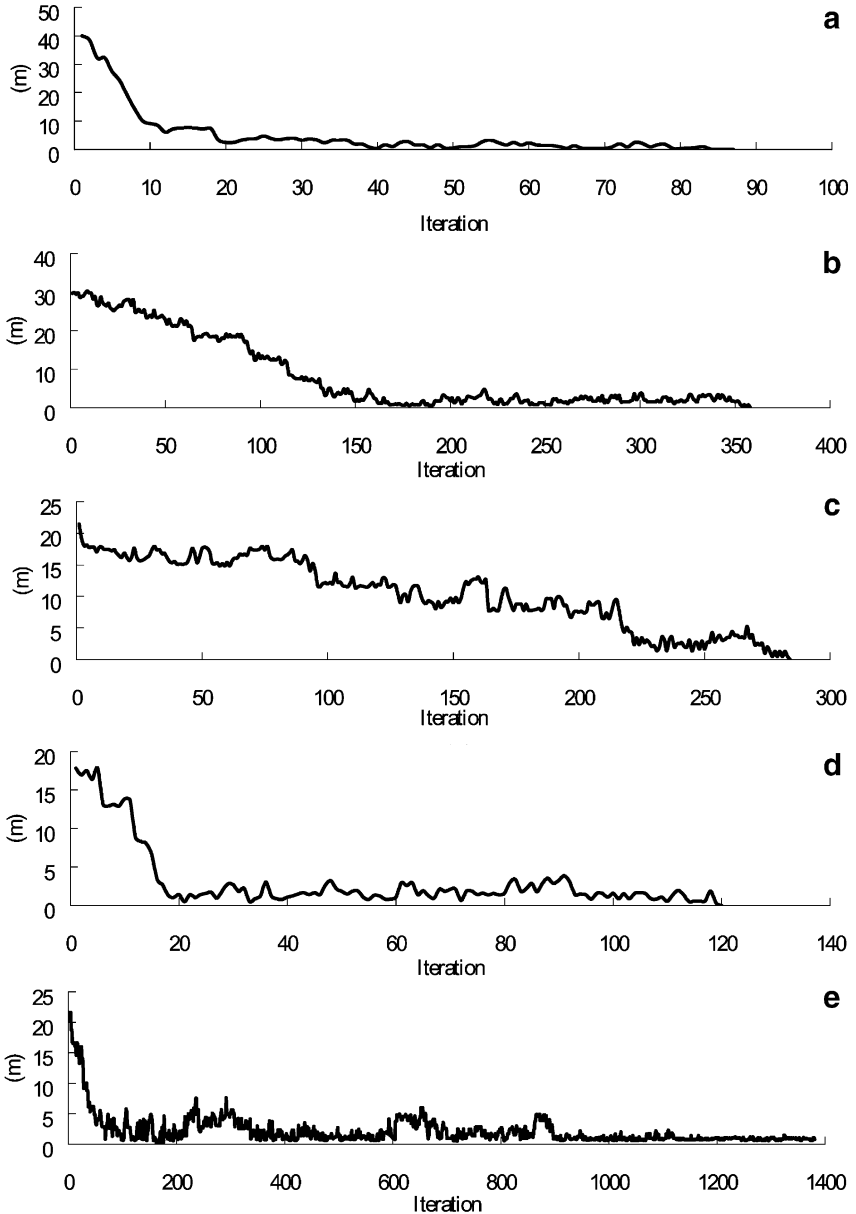

Fig. 6

The value of objective function for $\mathbf{a}$ the first trial; $\mathbf{b}$ the second trial; $\mathbf{c}$ the third trial; $\mathbf{d}$ the fourth trial; e the fifth trial

Table 3

The results of different trials (unit: $\mathrm{m}$ )

\begin{tabular}{|c|c|c|c|c|c|}
\hline & Trial 1 & Trial 2 & Trial 3 & Trial 4 & Trial 5 \\
\hline Initial objective function value & 39.97 & 29.70 & 21.46 & 17.85 & 21.62 \\
\hline Objective function value of best-recorded solution & 0 & 0 & 0 & 0 & 0.09 \\
\hline Find the global optimum & Yes & Yes & Yes & Yes & No \\
\hline
\end{tabular}

four times out of the five trials, as summarized in Table 3. In the failed instance, the zone two cells different from the global optimum is a local optimum, as shown in Fig. 5f. Figure 6 plots the values of the objective function values during the iterations in the five trials. Furthermore, the optimal simulated head, identical to the observed head, was reached in all successful trials. As an example, Fig. 7 displays the initial hydraulic head, the optimal head and the observed head in trial one. Thus, the results verified that applying SA with the SD method efficiently optimizes the spatial distribution of hydraulic conductivity.

\section{Scenario 2}

In a more realistic scenario, the spatial distribution of hydraulic conductivity and numbers of its type are all unknown. Five trials are performed. Two hydraulic conductivity zones are randomly selected in each run to initiate the optimization, as shown in Fig. 8. Table 4 shows the results. Figure 9 presents the values of the objective function in the five trials. The number of zones in a trail is less than the number of zones in global optimal zoning. Therefore, the values of the objective function converged only to a local optimum. 

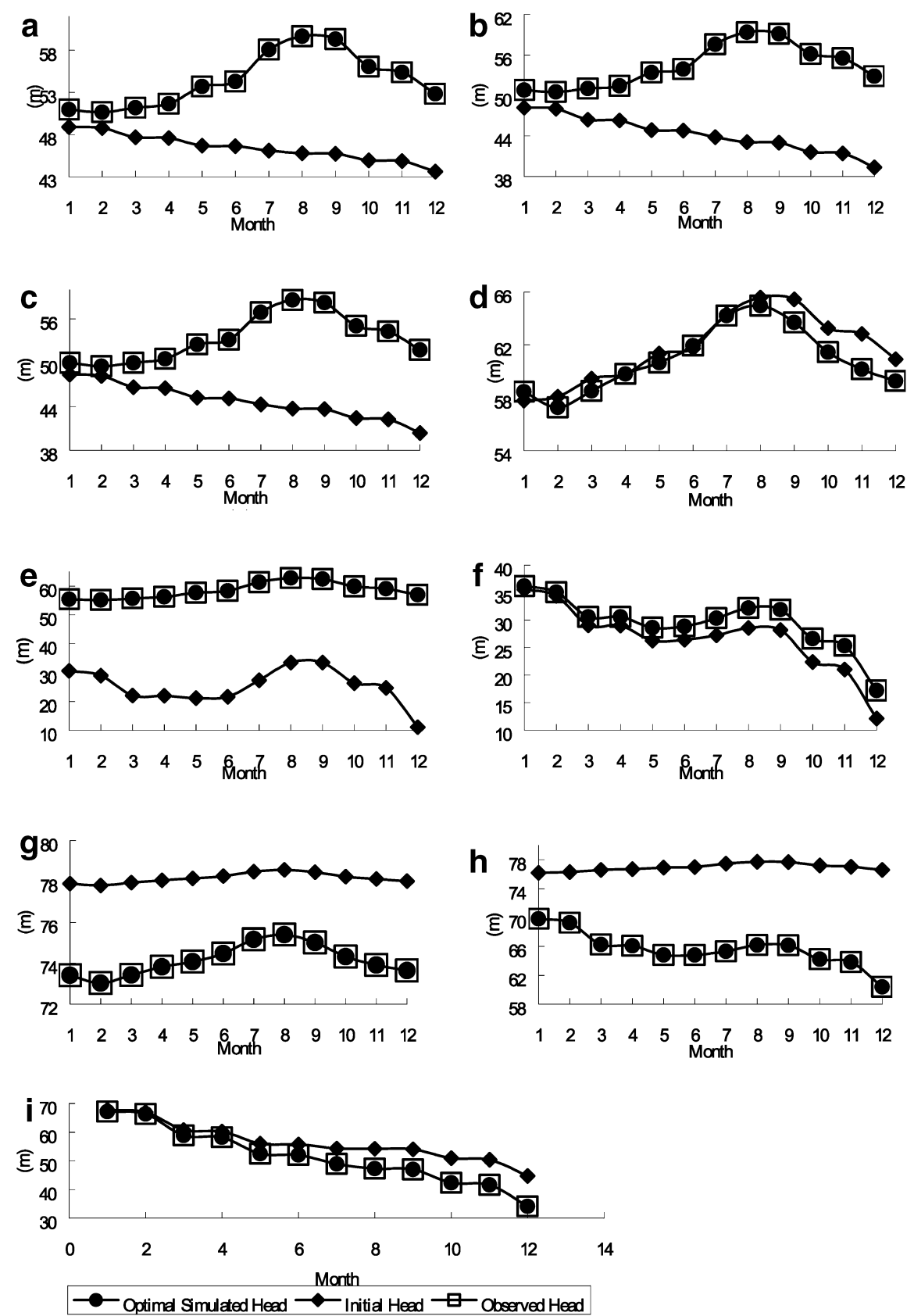

Fig. 7

Simulated and observed head of a well I, b well II, c well III, d well IV, e well V, $\mathbf{f}$ well VI $\mathbf{g}$ well VII $\mathbf{h}$ well VIII, $\mathbf{i}$ well IX

Trials that involved three hydraulic conductivity zones were performed, as shown in Fig. 10. Table 4 shows the results. The global optimum was found four times out of the five trials. In the trial that failed, a local optimum was just three grids different from the global optimum, as shown in Fig. 10f. Then, another five trials with four hydraulic conductivity zones were performed, as shown in Fig. 11. Table 4 shows the results. The global optimum was found three out of five times. In the two failed trials, as shown in Fig. 11f, g, local optima were found. Five trials with five hydraulic conductivity zones were performed, as shown in Fig. 12. Table 4 shows the results. The global optimum was found four out of five times. In the failed trial, as shown in Fig. 12f, zoning and hydraulic conductivity were incorrect. Figure 13 shows the values of the objective function for the five trials. As an example of scenario 2, Fig. 14 shows the initial hydraulic head, the optimal head, and the observed head in trial one of five initial hydraulic conductivity zones. The results conformed that applying SA and SD method can optimize the spatial distribution of hydraulic conductivity in more realistic cases.

Moreover, the global optimal spatial distributions of hydraulic conductivity were found not only with three zones, but also with initially given four and five zones, as illustrated in Table 4. Scenario 2 is feasible and applies to situations lacking hydrogeologic observation wells. However, the efficiency of optimization is maintained only if the number of hydraulic conductivity zones is not increased further when the value of the 

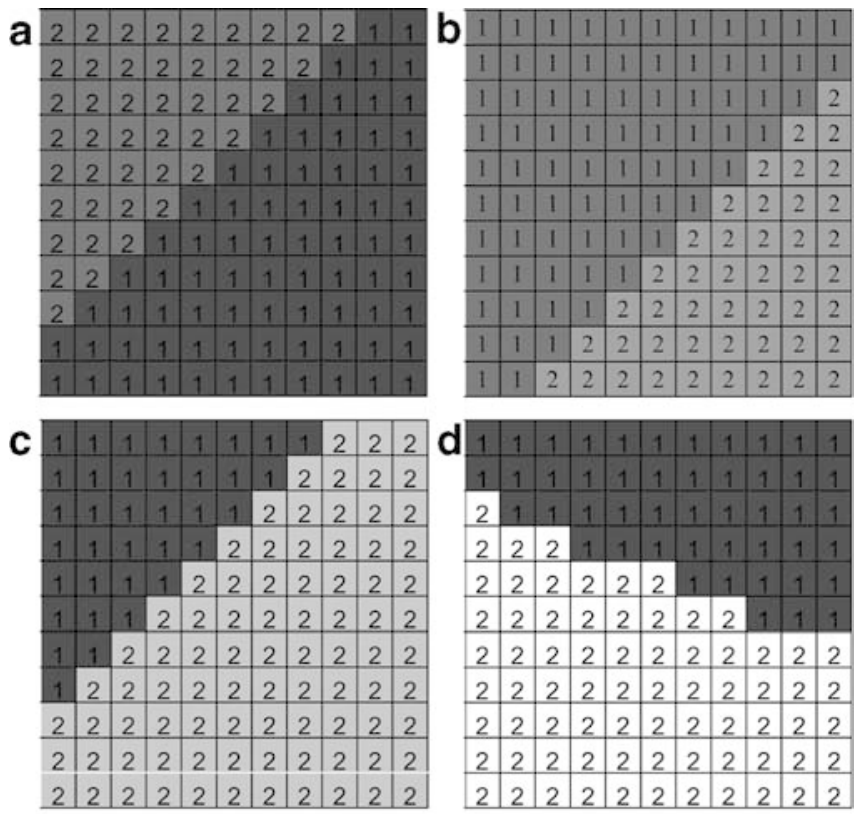
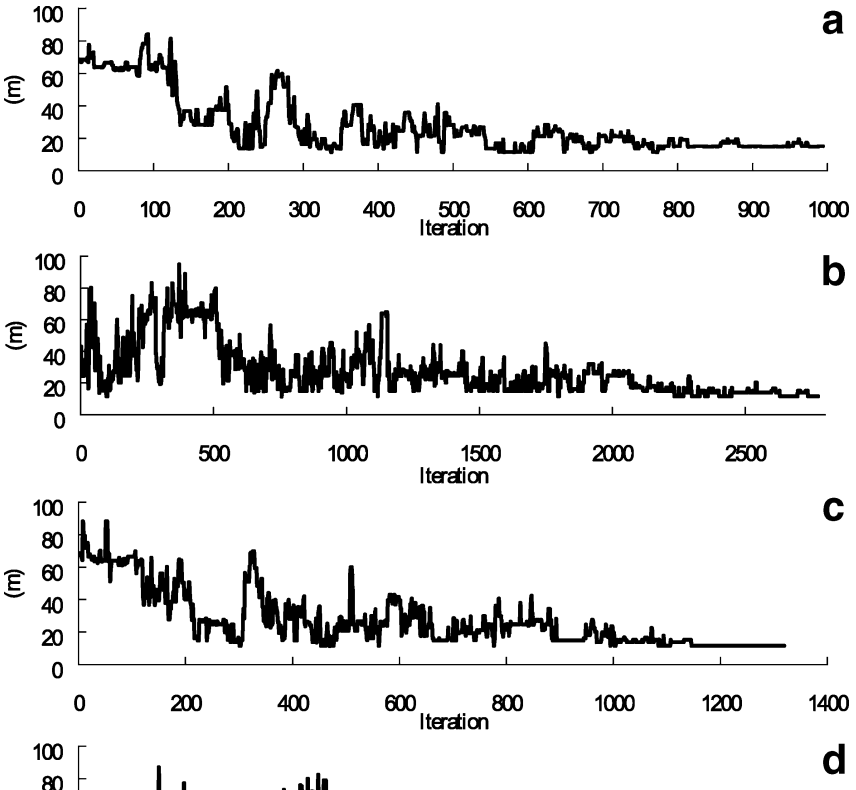

\section{है}
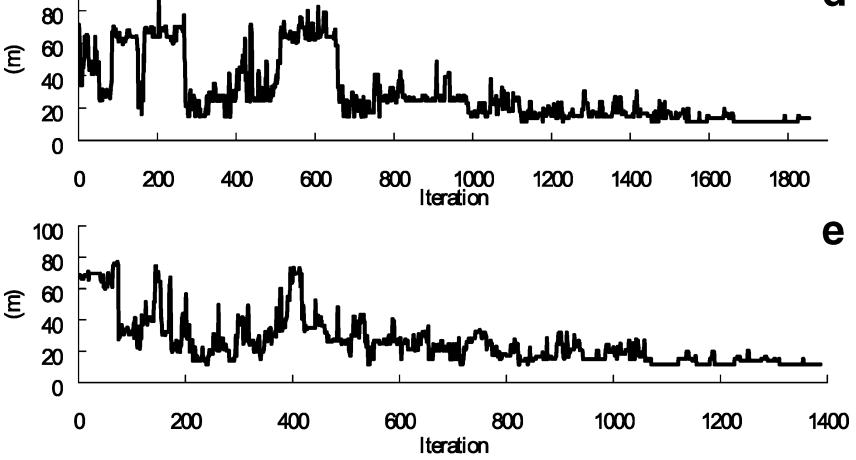

Fig. 9

The value of objective function for $\mathbf{a}$ the first trial; $\mathbf{b}$ the second trial; $\mathbf{c}$ the third trial; $\mathbf{d}$ the fourth trial; e the fifth trial

Table 4

The results of different zonation (unit: $\mathrm{m}$ )

\begin{tabular}{|c|c|c|c|c|c|c|}
\hline Initial type & & Trial 1 & Trial 2 & Trial 3 & Trial 4 & Trial 5 \\
\hline \multirow[t]{4}{*}{ Two zones } & Initial objective function value & 67.85 & 35.11 & 68.59 & 71.36 & 68.89 \\
\hline & $\begin{array}{l}\text { Objective function value of best recorded } \\
\text { solution }\end{array}$ & 11.66 & 11.66 & 11.66 & 11.66 & 11.66 \\
\hline & Find the global optimum & No & No & No & No & No \\
\hline & Find the correct hydraulic conductivity & No & No & No & No & No \\
\hline \multirow[t]{4}{*}{ Three zones } & Initial objective function value & 82.51 & 43.02 & 66.73 & 104.64 & 100.28 \\
\hline & $\begin{array}{l}\text { Objective function value of best recorded } \\
\text { solution }\end{array}$ & 0 & 0 & 0.72 & 0 & 0 \\
\hline & Find the global optimum & Yes & Yes & No & Yes & Yes \\
\hline & Find the correct hydraulic conductivity & Yes & Yes & Yes & Yes & Yes \\
\hline \multirow[t]{4}{*}{ Four zones } & Initial objective function value & 73.07 & 99.14 & 48.55 & 77.33 & 89.49 \\
\hline & $\begin{array}{l}\text { Objective function value of best recorded } \\
\text { solution }\end{array}$ & 0 & 0.34 & 0 & 0 & 0.18 \\
\hline & Find the global optimum & Yes & Yes & Yes & Yes & No \\
\hline & Find the correct hydraulic conductivity & Yes & No & Yes & Yes & Yes \\
\hline \multirow{4}{*}{ Five zones } & Initial objective function value & 55.45 & 64.24 & 51.93 & 140.12 & 99.71 \\
\hline & $\begin{array}{l}\text { Objective function value of best recorded } \\
\text { solution }\end{array}$ & 0 & 0 & 0 & 3.03 & 0 \\
\hline & Find the global optimum & Yes & Yes & Yes & No & Yes \\
\hline & Find the correct hydraulic conductivity & Yes & Yes & Yes & No & Yes \\
\hline
\end{tabular}



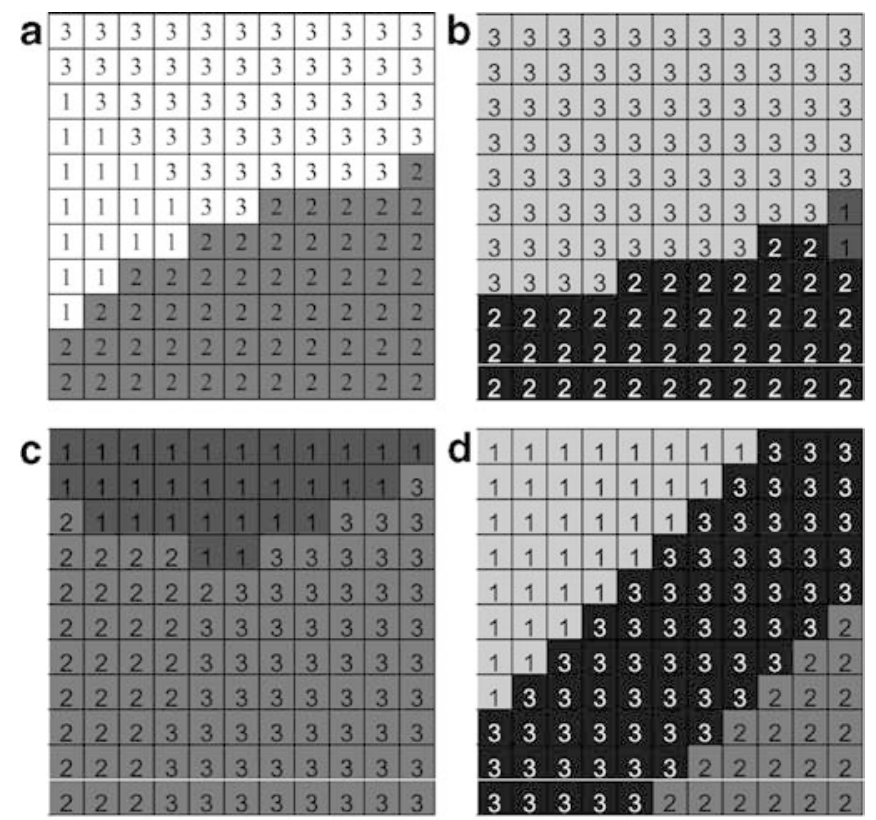

e
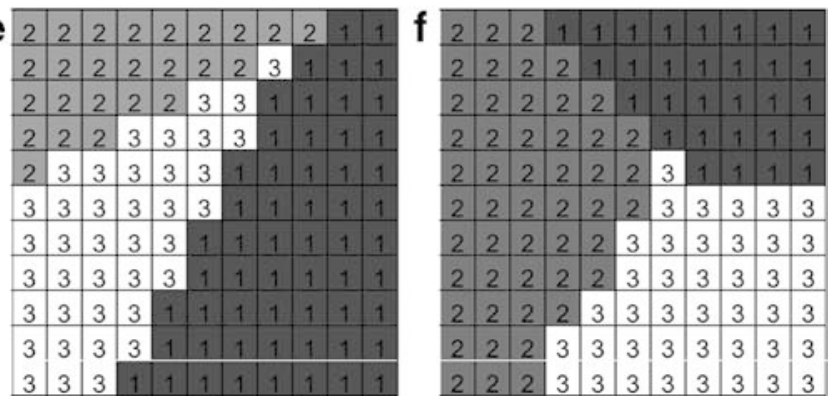

Fig. 10

a The initial zonation of the first trial; $\mathbf{b}$ the initial zonation of the second trial; $\mathbf{c}$ the initial zonation of the third trial; $\mathbf{d}$ the initial zonation of the fourth trial; $e$ the initial zonation of the fifth trial; $f$ the final zonation of the fifth trial

objective function does not continue to improve. Trial and error is suggested to gradually increase the number of types of hydraulic conductivity. When the number of zones in a trial is equal to or larger than the number of zones in the global optimal zoning, the global optimal spatial distribution of hydraulic conductivity will be found.

\section{Comparisons of the optimal zoning method and MODFLOW}

The newer version of MODFLOW built in an inverse solver with the Gauss-Marquardt-Levenberg method of nonlinear parameter estimation is also performed in the case of the initial solutions of five trials with three zones (Fig. 8) in scenario 2. Table 5 shows the differences between simulated and observed hydraulic heads at the nine observed wells by using the optimal zoning method and the newer version of MODFLOW. The simulated results illustrated that the optimal zoning method simulated more accurate hydraulic heads than the MODFLOW simulation did. The proposed optimal method successfully identifies the spatial distribution of hydraulic conductivity four times out of five trials because of automatically adjustment of both zonation and hydraulic conductivity using simulated annealing and short distance methods. Moreover, in this
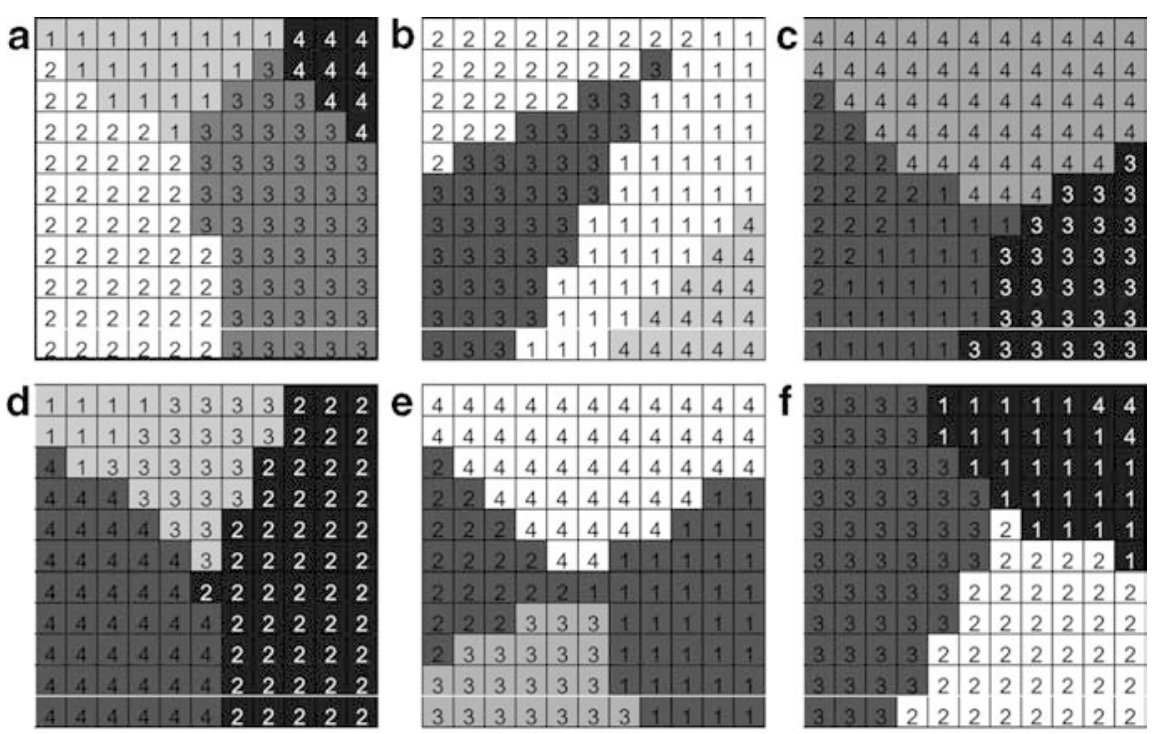

g

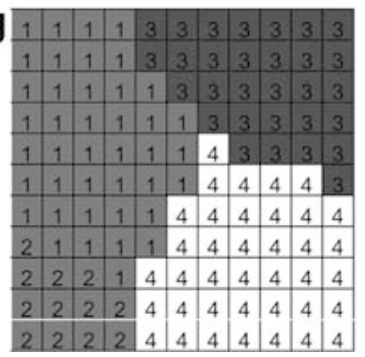

Fig. 11

a The initial zonation of the first trial; $\mathbf{b}$ the initial zonation of the second trial; $\mathbf{c}$ the initial zonation of the third trial; $\mathbf{d}$ the initial zonation of the fourth trial; e the initial zonation of the fifth trial; $f$ the final zonation of the second trial; $g$ the final zonation of the fifth trial 

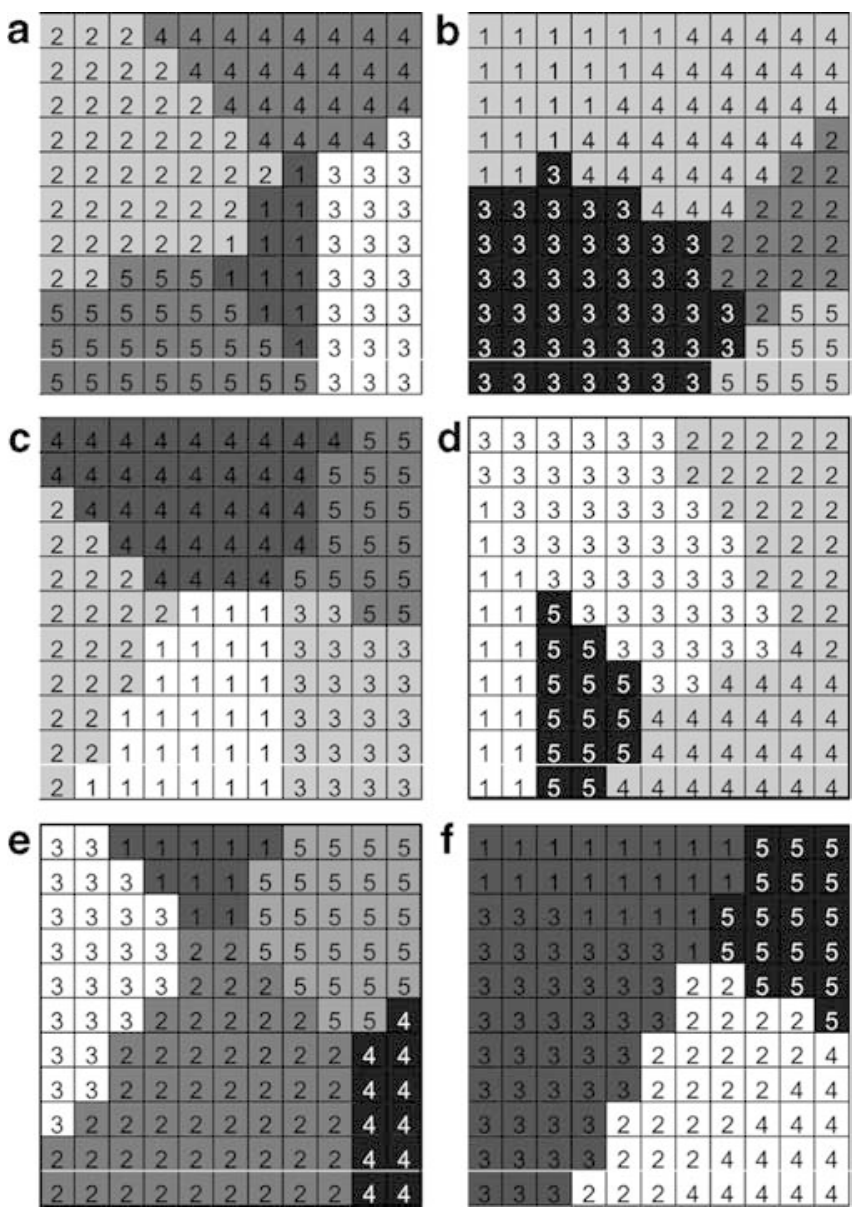

Fig. 12

a The initial zonation of the first trial; $\mathbf{b}$ the initial zonation of the second trial; $\mathbf{c}$ the initial zonation of the third trial; $\mathbf{d}$ the initial zonation of the fourth trial; $e$ the initial zonation of the fifth trial; $f$ the final zonation of the fourth trial

case the optimal zoning method is more promising to reach a global optimum of simulating hydraulic heads than the MODFLOW with the Gauss-Marquardt-Levenberg method. The newer version of MODFLOW with the GaussMarquardt-Levenberg method of nonlinear parameter estimation may need much more trial times with different initial zonations to delineate the spatial distribution of hydraulic conductivity and to simulate accurate hydraulic heads. Meanwhile, the computational time, running in a personal computer with Pentium III $733 \mathrm{MHz}$, are 15 and 130 s per each trial for running the MODFLOW and the optimal zoning method.

\section{Conclusions}

This study presented a novel procedure to apply MODFOLW, combined with SA and SD method, to optimize the spatial distribution of hydraulic conductivity. The
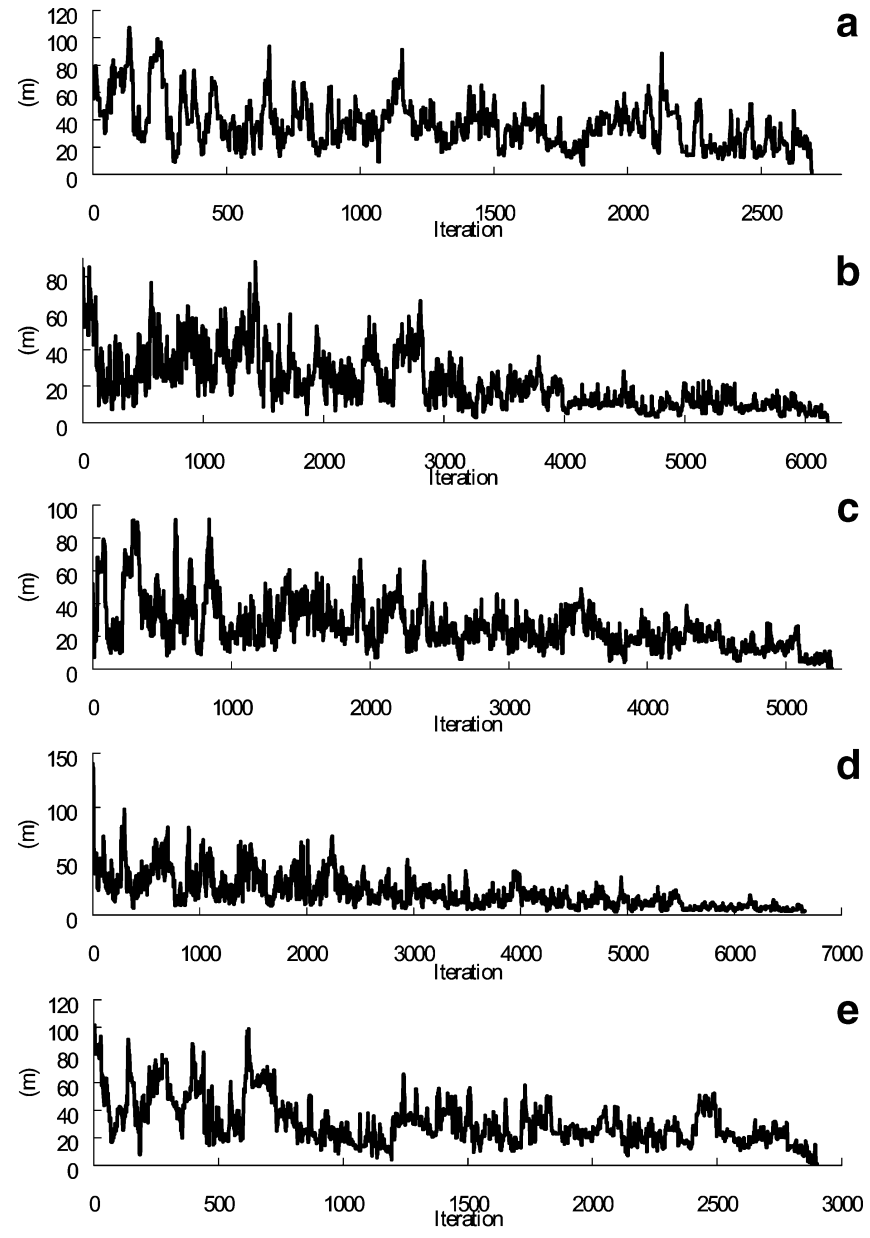

Fig. 13

The value of objective function for a the first trial; $\mathbf{b}$ the second trial; c the third trial; $\mathbf{d}$ the fourth trial; e the fifth trial

hydraulic head was simulated just as an observed head in a hypothetical case, and the zoning of hydraulic conductivity was globally optimized. This new procedure not only improves the efficiency of optimization, but also increases the probability of finding the global optimum, minimizing errors in simulating in two scenarios. In the first scenario, the hydraulic conductivity over the site was known; in the second it was not. The success rate exceeds half not only in scenario 1, but also in scenario 2. Moreover, the proposed method can efficiently delineate hydrological zonation with more accurate simulated hydraulic heads than the inverse solver in MODFLOW do. The proposed procedure in this study can be expected to be reasonably applied to a real complex site, resulting in more accurate simulations of groundwater than provided by other methods. However, the SD method may fail to determine hydrological zonations for strongly anisotropic even sinuous patterns that are common in geology. Therefore, advanced classification techniques should be integrated into the proposed method to solve this weakness for further study. 

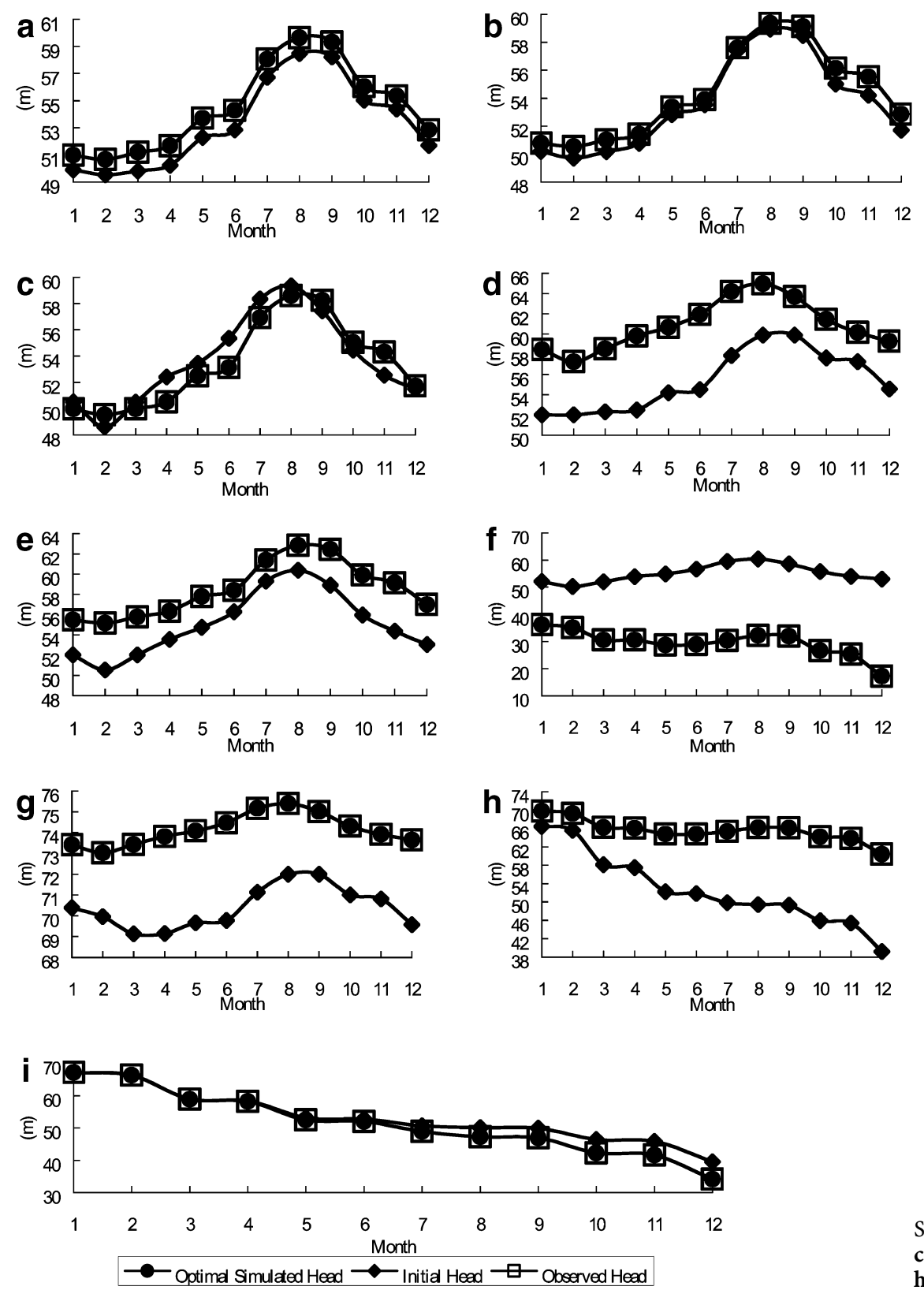

Fig. 14

Simulated and observed head of a well I, $\mathbf{b}$ well II, c well III, d well IV, e well V, f well VI, $\mathbf{g}$ well VII, h well VIII, i well IX

Table 5

Mean differences of simulated and observed hydraulic heads (unit: m). Mean difference: difference between simulated and observed heads/ number of well

\begin{tabular}{|c|c|c|c|c|c|c|}
\hline Method & Trial 1 & Trial 2 & Trial 3 & Trial 4 & Trial 5 & Mean \\
\hline The optimal zoning method & 0 & 0 & 0.08 & 0 & 0 & 0.02 \\
\hline The MODFLOW with an inverse solver & 6.73 & 6.43 & 5.73 & 5.77 & 6.50 & 6.23 \\
\hline
\end{tabular}

\section{References}

Abdulla FA, Al-Khatib MA, Al-Ghazzawi ZD (2000) Development of groundwater modeling for the AZROQ basin. Environ Geol 40(1/2):11-18

Detwiler RL, Mehl S, Rajaram H, Cheung WW (2002) Comparison of an algebraic multigrid algorithm to two iterative solvers used for modeling ground water flow and transport. Ground Water 40(3):267-272
Deutsch CV, Journel AG (1992) Geostatistical software library and user's guide. Oxford University Press, New York

Dougherty DE, Marryott RA (1991) Optimal groundwater management: 1. simulated annealing. Water Resour Res 27(10):2493-2508

Ella VB, Melvin SW, Kanwar RS, Jones LC, Horton R (2002) Inverse three-dimensional groundwater modeling using the finitedifference method for recharge estimation in a glacial till aquitard. Trans Am Soc Agric Eng 45(3):703-71 
Kaufman L, Rousseeuw PJ (1990) Finding groups in data. Wiley, New York

Kirkpatrick S (1984) Optimization by simulated annealing: quantitative studies. J Statistic Phys 34(5/6):975-986

Kirkpatrick S, Gelatt CD, Vecchi MP (1983) Optimization by simulated annealing. Science 220:671-680

Lin YP, Chang TK, Teng TP (2001a) Characterization soil lead by comparing of sequential Gaussian simulation, simulated annealing simulation and kriging methods. Environ Geol 41(1/ 2):189-199

Lin YP, Tan YC, Rouhani S (2001b) Identifying spatial characteristics of transmissivity using simulated annealing and kriging methods. Environ Geol 41:200-208

Majumdar PK, Ghosh NC, Chakravorty B (2002) Analysis of arsenic-contaminated groundwater domain in the Nadia district of West Bengal (India). Hydrol Sci, J Sci Hydrol 47:S55-S66

McDonald MG, Harbaugh AW (1988) A modular three-dimensional finite-difference ground-water flow model. Techniques of Water Resources Investigations, 06-A1. US Geological Survey Metropolis N, Rosenbluth A, Rosenbluth M, Teller A, Teller E, Chem J (1953) Equation of state calculations by fast computation machines. Physics 21:1087-1092

Mikake S, Yoshida H, Koide K, Yanagizawa K, Ogata N, Maekawa K (2000) Methodology development for modeling heteroge- neous conductivity fields for a sandstone type uranium deposit, central Japan. Eng Geol 56:185-195

Roth C, Chiles JP, De Fouquet C (1998) Combing geostatistics and flow simulators to identify transmissivity. Adv Water Resour 21(7):555-565

Sophocleous MA, Koelliker JK, Govindaraju RS, Birdie T, Ramireddygari SR, Perkins SP (1999) Integrated numerical modeling for basin-wide water management: the case of the Rattlesnake Creek basin in south-central Kansas. J Hydrol 214:179-196 Tung CP, Chou CA (2002) Application of tabu search to groundwater parameter zonation. J Am Water Resour Assoc 38(4):1115-1126

Wang PP, Zheng C (1998) An efficient approach for successively perturbed groundwater models. Adv Water Resour 21:499-508 Wen XH, Capilla JE, Deutsch CV, Gomez-Hernandez JJ, Cullick AS (1999) A program to create permeability fields that honor single-phase flow rate and pressure data. Comput Geosci 25:217-230

Wingle WL, Poeter EP, McKenna SA (1999) UNCERT: geostatistics, uncertainty analysis and visualization software applied to groundwater flow and contaminant transport modeling. Comput Geosci 25:365-376 